\section{REVISTA}

EPUGACIÓN

Revista Educación

ISSN: 0379-7082

ISSN: 2215-2644

revedu@gmail.com

Universidad de Costa Rica

Costa Rica

\title{
Juegos serios para potenciar la adquisición de competencias digitales en la formación del profesorado
}

Sandí Delgado, Juan Carlos; Sanz, Cecilia Verónica

Juegos serios para potenciar la adquisición de competencias digitales en la formación del profesorado

Revista Educación, vol. 44, núm. 1, 2020

Universidad de Costa Rica, Costa Rica

Disponible en: http://www.redalyc.org/articulo.oa?id=44060092019

DOI: https://doi.org/10.15517/revedu.v44i1.37228

Esta obra está bajo una Licencia Creative Commons Atribución-NoComercial-SinDerivar 3.0 Internacional. 


\title{
Juegos serios para potenciar la adquisición de competencias digitales en la formación del profesorado
}

\author{
Juan Carlos Sandi Delgado \\ Universidad de Costa Rica, Costa Rica \\ juan.sandidelgado@ucr.ac.cr \\ iD http://orcid.org/0000-0003-3932-3045 \\ Cecilia Verónica Sanz \\ Universidad Nacional de La Plata (UNLP), Argentina \\ csanz@lidi.info.unlp.edu.ar
}

Serious Games to Enhance Digital Competencies Acquisition for Training Faculty

(DD http://orcid.org/0000-0002-9471-0008

\author{
DOI: https://doi.org/10.15517/revedu.v44i1.37228 \\ Redalyc: http://www.redalyc.org/articulo.oa?id=44060092019
}

Recepción: 13 Junio 2019

Aprobación: 18 Octubre 2019

\section{Resumen:}

El objetivo principal de esta investigación consiste en indagar y analizar las fortalezas de los juegos serios para potenciar la adquisición de competencias digitales en el profesorado. A través de una revisión bibliográfica se desarrolla un análisis comparativo de las principales características de una serie de juegos serios elegidos según los criterios de análisis: a) País de origen del juego e idiomas que soporta, $b$ ) Intensión pedagógica, $c$ ) Individual o interacción con otros, d) Guías/ayudas disponibles, e) Destinatarios del juego, $f$ ) Resultados de utilización, $g$ ) Estrategias de ludificación. En la selección, se consideraron juegos serios que hayan sido utilizados para formar competencias digitales en el profesorado en educación superior, durante la última década (2009 al 2019 inclusive). Entre los principales resultados, se identificó que los juegos serios facilitan cambios de actitud y comportamiento, posibilitan la innovación pedagógica, potencian la interacción con otros, promueven la investigación, facilitan la adquisición de competencias y habilidades tecnológicas, incentivan el respecto y concientización social, ético y legal del uso de las TIC. Se concluye que, los juegos serios además de ser innovadores, interactivos y promover la investigación, son beneficiosos para potenciar la formación de competencias digitales en docentes en diferentes áreas del conocimiento. Como línea de trabajo futuro, se plantea la elaboración de una propuesta de aplicación de un juego serio con el propósito de potenciar la adquisición y/o formación de competencias digitales por parte del personal docente a nivel de educación superior.

Palabras clave: Tecnologías de la información y la comunicación (TIC), Competencias digitales, Formación docente, Profesorado, Educación superior.

\section{ABstract:}

This study investigates and analyzes the strengths of educational games to enhance the acquisition of digital skills capabilities among faculty. Through a bibliographic review, a comparative analysis of the main characteristics of a series of educational games selected according to the analysis criteria used for this study: a) Country of origin of the game and languages it supports, b) Pedagogical intent, c) Individual or interaction with others, d) Guides/aids available, e) Recipients of the game, f) Results, and g) Gaming strategies. The games that were chosen had been used previously during the last decade (2009-2019) for training university faculty. Findings reveal that educational games facilitate changes in attitude and behavior, enable pedagogical innovation, enhance interaction with others, promote research, facilitate the acquisition of technological competencies and skills, and encourage respect and promotes awareness about social, ethical and legal use of ICTs. Aside from being innovative and interactive, games promote research and help maximize digital skills acquisition among teachers in different areas of knowledge.

KEYWORDS: Information and communication technologies (ICTs), Digital Competencies, Teacher-training, Teachereducation, Faculty, Higher Education. 


\section{INTRODUCCIÓN}

A razón del rápido avance de las Tecnologías de la Información y la Comunicación (TIC) y de algunas herramientas tecnológicas, el proceso formativo del profesorado universitario relacionado con habilidades y competencias TIC, se ha vuelto un reto y un requerimiento importante de atender en las Instituciones de Educación Superior (IES), porque las exigencias actuales a nivel educativo son mayores, donde se demandan currículos innovadores, creativos y flexibles que permitan potenciar a nivel del estudiantado y el profesorado, capacidades digitales conforme a los requerimientos de hoy de la sociedad, con el propósito de generar procesos formativos capaces de impulsar en el profesorado la adquisición de conocimientos significativos que les permitan el desarrollo de diferentes capacidades y la apropiación de las tecnológicas digitales, de sus lenguajes y posibilidades (Sandí y Sanz, 2018a). Al respecto, el investigador (Wartenweiler, 2018) agrega que los juegos serios desempeñan un rol protagónico a nivel educativo, debido a que en la actualidad se ha iniciado con su utilización para potenciar procesos de enseñanza y aprendizaje.

Otros investigadores (Connolly, Boyle, MacArthur, Hainey y Boyle, 2012) indican que han sido creados con el propósito de emplearse para fines educativos, la formación y adquisición de competencias, debido a que su objetivo caracterizante se enfoca al aprendizaje, formación de competencias digitales, cambios de actitud y comportamiento. Ahora bien, actualmente hace falta explorar y profundizar en las oportunidades que podrían brindar los juegos serios en la formación del personal docente, con el propósito de acercarlos a las TIC y a la adquisición de competencias digitales.

En España, (Romero y Turpo, 2012) a través de una investigación bibliográfica observaron en los juegos serios fortalezas para la formación en competencias digitales. Además, se refieren a las oportunidades para la formación de habilidades comunicativas, colaborativas, sociales y culturales. Igualmente, en España (Guenaga, Eguíluz, Rayón y Quevedo, 2015) utilizaron un juego serio para desarrollar y evaluar competencias del trabajo en equipo por parte del profesorado.

Con base en lo expuesto, este artículo ostenta una revisión vinculada con indagar fortalezas las cuales ofrecen los juegos serios al profesorado en relación con la formación de competencias digitales, con la intención de facilitar el uso y apropiación de las tecnologías digitales en el ámbito educativo, específicamente, a nivel de educación superior. Así, este trabajo pretende evidenciar las fortalezas de los juegos serios, estas servirán como referente para una investigación futura en la cual se pueda proponer e implementar una propuesta de formación docente a través de la utilización de juegos serios.

El artículo se organiza y presenta así: el apartado 2 aborda términos claves relacionados (el concepto de competencias tecnológicas, simulación y clasificación de los juegos (advergames, edutainment, simulación y la definición de juegos serios). El apartado 3 corresponde a la metodología de investigación utilizada, el apartado 4 ostenta el análisis de juegos serios con respecto a sus oportunidades para la formación de competencias digitales, donde la sección 4.1, se definen criterios de análisis, en la sección 4.2 presenta la selección de los juegos serios y, seguido, la sección 4.3 destaca el análisis global efectuado a los juegos serios seleccionados. El apartado 5 resume las principales conclusiones y líneas futuras de trabajo.

\section{TÉrminos CLAVES RELACIONADOS}

En esta sección se abordan las definiciones de competencias tecnológicas, simulación y la clasificación de los juegos (advergames, edutainment, simulación y juegos serios. Lo cual permite contextualizar el análisis que se desarrolla en forma posterior acerca de algunas fortalezas proporcionadas por los juegos serios en educación superior en relación a habilidades y/o competencias digitales del personal docente. Esta sección permite brindar respuesta a las preguntas de investigación: RQ1, RQ2 y RQ3 (descritas a detalle en el apartado 3 correspondiente a la metodología). 


\subsection{Competencias tecnológicas (RQ1)}

El concepto de competencias tecnológicas ${ }^{[1]}$ puede ser conocido por diferentes terminologías dependiendo la institución, organización o investigador que las releva. Por ejemplo, se les conoce como alfabetización digital, alfabetización en TIC, competencias TIC y competencias digitales (Cruz, 2018; Prendes, Gutiérrez y Martínez, 2018; Rodríguez, Raso y Ruiz, 2019). Las competencias comprenden variedad de unidades cognitivas, las cuales se fundamentan en habilidades (pedagógicas, tecnológicas) y componentes afectivos: conocimientos, valores, ética, actitudes; apertura, recepción, persistencia y aprender a partir de errores (Cruz, Sandí y Víquez, 2017; Sandí y Cruz, 2016, 2017a). Al respecto, Claro et al. (2018) afirman que la alfabetización digital del profesorado incluye el uso correcto y crítico de las TIC en la educación con el propósito de proponer soluciones a inconvenientes cognitivos los cuales surgen a diario, además, debe ser capaz de servir de mediador entre la tecnología y los procesos formativos.

Por tal razón, es recomendable que las IES incluyan dentro de sus objetivos organizaciones y de gestión, la formación y actualización constante del profesorado en tecnologías, con el propósito de formar personas digitales responsables, informadas, actualizadas y comprometidas en la actualización constante en competencias tecnológicas, con el propósito de ser facilitadores de procesos formativos acordes a las demandas de la sociedad actual (Choi, Cristol y Gimbert, 2018). Para este estudio, se hace referencia al concepto de competencias tecnológicas brindado por Sandí, Sanz y Lovos (2018):

Las competencias tecnológicas pueden definirse como un conjunto de saberes relacionados con el uso y dominio de las tecnologías digitales y otras áreas del conocimiento relacionadas, que a través de diferentes actitudes y valores permiten a las personas el saber ser, es decir, desarrollar un comportamiento propio ante un evento con el fin de potenciar el mejoramiento personal y/o profesional. A su vez, involucra la habilidad de adquirir nuevos conocimientos y la destreza para saber hacer y resolver de la mejor forma las actividades relacionas al entorno en el que se desenvuelve (pp. 24-25).

\subsection{Clasificación de los juegos (RQ2)}

En esta sección se aborda y describe brevemente algunas de las categorías en las que se podría clasificar los juegos, con el propósito de identificar su objetivo caracterizante.

\subsubsection{Advergames}

El término advergames se refiere a aquellos juegos cuyo objetivo caracterizante es la mercadotecnia (marketing), es decir, desarrollados específicamente para ser jugados por diversión y entregar mensajes publicitarios vinculados con marcas o productos en específico (Lee y Cho, 2017; Roettl, Waiguny y Terlutter, 2016)

\subsubsection{Edutainment}

El término edutainment (entretenimiento educativo) hace referencia a la combinación de la formación con el entretenimiento, en otras palabras, son juegos que combinan contenidos educativos con elementos lúdicos; caracterizados principalmente por ser utilizados para la asimilación de conceptos e instrucciones, en los cuales se práctica de forma reiterada algún tipo de actividad (de forma de entretenimiento educativo). Es decir, el jugador no requiere necesariamente de la aplicación de conocimientos, analizar crítica y reflexivamente, además, no se ejecuta algún tipo de evaluación de los aprendizajes (feedback) (Charsky, 2010; Ma y Oikonomou, 2017). 


\subsubsection{La simulación (RQ3)}

El concepto de simulación ha sido definido en diferentes investigaciones (Angelini, García y Martínez, 2017; Dávila, 2014), en las cuales se coincide en su capacidad para potencializar habilidades y competencias a través del juego en diferentes áreas del conocimiento. En este sentido, la simulación podría definirse como “... un recurso electrónico que permite el diseño de ambientes simulados de aprendizaje, en los cuales se potencian diferentes habilidades cognitivas del estudiantado sin poner en riesgo su integridad física o emocional" (Sandí y Cruz, 2017a, p.4).

La simulación y el juego son metodologías que a través de diferentes tipos de escenarios posibilitan la adquisición de diferentes conocimientos en contextos reales, es decir, conocimientos tecnológicos, pedagógicos, lingüísticos, de comunicación, liderazgo, trabajo en equipo, entre otros (Angelini et al., 2017). En otras palabras, la simulación podría potenciar en el profesorado la adquisición de nuevas habilidades en diferentes áreas del conocimiento, a través de la integración de conceptos especializados y competencias profesionales, en este caso en específico, la formación de competencias digitales (Angelini et al., 2017; Dávila, 2014).

Actualmente, la simulación se ha convertido en un recurso tecnológico estratégico a nivel educativo, al permitir al profesorado potenciar la mejora continua de los procesos formativos a través de su implementación, es decir, la simulación posibilita en el estudiantado habilidades que les permiten relacionarse con otros de una forma más fácil, desarrollar mayor tolerancia hacia la frustración, potenciar la motivación por alcanzar objetivos y la resolución de problemas (López, 2016; Sandí y Cruz, 2017a).

\subsubsection{Juegos serios (RQ4)}

En 1970 se dio a conocer por primera vez el término de juegos serios, el cual fue introducido por el investigador Abt (1970), cuyas investigaciones estaban relacionadas con indagar sus posibilidades para instrucción, formación, educación, motivación y en la formación de nuevas competencias y habilidades (Sandí y Sanz, 2018a).

Los investigadores Michael y Chen (2006), señalan que los juegos serios van más allá del hecho de entretener y la diversión, sino que pueden ser utilizados para favorecer cambios en la actitud, en el comportamiento y, potenciar los procesos formativos, conservando sus características relacionadas con la diversión y el entretenimiento.

Los juegos serios podrían ser incorporados en los procesos formativos, a través de simulaciones, mundos virtuales, videojuegos o realidad aumentada (Sandí y Cruz, 2018). Los juegos serios poseen un propósito variado; se han utilizado para crear conocimientos, cambios de actitud, generación de emociones, aplicables en la salud, el entrenamiento, la formación y la educación (López, 2016; Sandí y Bazán, 2019).

En suma, los juegos serios buscan potenciar el aprendizaje, la estimulación del pensamiento crítico, el entrenamiento, la alfabetización digital, cambios de actitud y generación de emociones, lo cual va más allá del componente lúdico propio de los juegos.

Los cuales podrían servir de mediadores y facilitadores en las IES para la configuración de procesos formativos innovadores (Padilla, Gutiérrez y Medina, 2011). Claro está, que estos juegos serios deben pasar por un proceso de reestructuración para ser adaptados acorde al propósito pedagógico propio que efectúa cada institución educativa. Lo anterior, representa una oportunidad para acercar al personal docente a las tecnologías, mediante el juego.

Si bien, los juegos serios podrían ser catalogados dentro de la categoría edutainment, se diferencian de los demás juegos, porque son pensados y desarrollados no solamente para el entretenimiento, el disfrute o la diversión directamente, sino que, esencialmente poseen un propósito educativo explícito y cuidadosamente 
pensado, además, incorporan estrategias de ludificación (medallas, ranking, insignias, puntajes, premiación, entre otros) (Ma y Oikonomou, 2017; Sandí y Sanz, 2018a).

\section{Metodología de investigación Utilizada}

La metodología se trabajó desde un enfoque cualitativo, mediante una revisión sistemática de bibliografía, en la cual se ha seguido el protocolo propuesto por Kitchenham et al. (2009), el plantea la siguiente estructura: a) Definición para la búsqueda: definir preguntas de investigación, el alcance de la revisión, definir criterios para la inclusión y exclusión de referencias, definir palabras claves y cadenas de búsquedas. b) Ejecución de la búsqueda: definir la selección de trabajos primarios y difusión de criterios de análisis. c) Discusión de los resultados: definir esquemas de caracterización y analizar los resultados. Protocolo que ha sido previamente utilizado en otras investigaciones (Cruz y Bazán, 2018; Cruz y Sandí, 2017; Sandí y Cruz, 2017b, Sandí y Espinoza, 2018) y ha resultado efectivo. Entre las principales decisiones tomadas en cuenta para llevar adelante el proceso de la investigación, acorde al protocolo propuesto, están:

\section{a) Definición para la búsqueda}

$\checkmark$ Preguntas de investigación: para alcanzar el objetivo del estudio, se definieron 6 preguntas de investigación (RQs), las cuales abarcaron aspectos conceptuales tales como: RQ1: ¿Cómo se define el término competencias tecnológicas?, RQ2: ¿Cómo se clasifican los juegos educativos?, RQ3: ¿Cómo se define el concepto de simulación?, RQ4: ¿Cómo se define el concepto de juegos serios?, RQ5: ¿Qué posibilidades ofrecen los juegos serios para adquirir competencias digitales en docentes? RQ 6 ¿Cuáles son las fortalezas que se identifican en los juegos serios para potenciar la formación y/o adquisición de competencias digitales en el profesorado?

$\checkmark$ Alcance de la revisión: Las RQs fueron determinadas con la intención de servir de guía para identificar características y fortalezas de los juegos serios para potenciar la formación y/o adquisición de competencias digitales en el profesorado a nivel de educación superior, acorde a los criterios de investigación que se detallan más adelante. La estrategia de búsqueda para la localización de los artículos relacionados con competencias tecnológicas y juegos serios consistió en indagar en diferentes bases de datos científicas y académicas (Martínez, 2016), tales como IEEE Xplore Digital Library, ScienceDirect y SCOPUS, se eligieron por su disponibilidad y acceso a la información requerida.

$\checkmark$ Criterios para la inclusión y exclusión de referencias: Se definieron los siguientes criterios de inclusión y exclusión:

- Criterios de inclusión: textos publicados en su versión completa, escritos en idioma español y/o inglés, publicados en los años 2009 al 2019 inclusive, relacionados con clasificación de los juegos, juegos serios, simulación y competencias tecnológicas, los cuales permitieran identificar experiencias y resultados de actividades que hayan utilizado juegos serios para formar competencias digitales.

- Criterios de exclusión: referencias no relacionadas con las RQs, escritos en otros idiomas distintos al español e inglés, investigaciones duplicadas de otras investigaciones, fuentes que no se logró tener acceso al documento completo.

\section{b) Palabras clave y cadenas de búsqueda:}

Se establecieron palabras claves y cadenas de búsqueda en idioma español e inglés acorde a los temas de interés de esta investigación. Palabras tales como: juegos serios (serious games), competencias digitales (digital 
competencies), características (Characteristics), simulación (simulation), entre otras. Entre las cadenas de búsqueda: Juegos serios y competencias digitales (serious games and digital competencies), Características y Juegos serios (Characteristics and serious games), entre otras.

\section{c) Ejecución de la búsqueda:}

Una vez localizados los trabajos primarios, se procedió a aplicar los criterios establecidos, dando como resultado los documentos empleados y citados en esta investigación. Para ello, se efectuó inicialmente una lectura rápida del título, resumen y palabras clave. Una vez obtenidos los documentos preseleccionados, se procedió a dar lectura completa y se aplicó nuevamente los criterios de inclusión y exclusión definidos.

\section{d) Discusión de los resultados:}

Para la discusión de resultados, fue necesario definir y describir los criterios de análisis (C), visibles en Tabla 1, correspondiente a la sección 4.1. Luego, se procede a elaborar la descripción de los juegos serios elegidos, esto acordes con las dimensiones estipuladas por el Ministerio de Educación de Chile (2006) (visibles en la sección 4.2).

\section{ANÁlisis DE JUEgos SERIOS ACORDE A SUS POSIBILIDADES PARA FORMAR COMPETENCIAS TECNOLÓgICAS EN EL PROFESORADO (RQ5)}

En esta sección se define un conjunto de criterios que se utilizarán en la elaboración del respectivo estudio vinculado con las posibilidades de los juegos serios para formar habilidades digitales en el personal docente. Seguido, se presentan los juegos elegidos para ser estudiados a la luz de los criterios establecidos previamente. Luego, se prepara una tabla resumen comparativa de las características identificadas en cada juego serios analizados y, por último, el análisis general de los juegos serios descritos. Esta sección permite brindar respuesta a la RQ5.

\subsection{Criterios definidos para el análisis de juegos serios}

A partir de la definición de juegos serios brindada en la sección 2.2.4, se definen criterios de análisis que serán aplicados al conjunto de juegos serios relevados, los cuales podrían posibilitar la adquisición y formación de habilidades y competencias digitales en el personal académico. Se debe indicar que, para la búsqueda de la muestra de los juegos serios, se tomó en consideración que dentro de sus objetivos se indicara claramente la formación de competencias digitales o el favorecimiento del desarrollo del pensamiento computacional.

Igualmente, fue importante tomar en cuenta que los juegos serios elegidos estén ligados o relacionados con al menos una de las dimensiones e indicadores de competencias que han sido presentadas por el Ministerio de Educación de Chile (2006), dimensiones adoptadas para este análisis.

Ahora bien, algunos de los juegos serios seleccionados están dirigidos a la población estudiantil, sin embargo, fue interesante elegirlos debido que potencian la formación de competencias digitales y podrían utilizarse con el profesorado. Igualmente, posibilitan al personal docente echar luz acerca de qué estrategias y dinámicas podrían motivar mayormente a la población estudiantil.

La Tabla 1, resume los criterios de análisis (C) establecidos para el estudio de los juegos serios elegidos. 
TABLA 1

Definición de criterios (C) para el análisis de juegos serios

\begin{tabular}{llll}
\hline Criterios de análisis & \\
\hline C1 & País de origen del juego e idiomas que soporta & C4 & Destinatarios del juego \\
\hline C2 & Intensión pedagógica & C5 & Resultados de utilización \\
\hline C3 & Guias layudas disponibles & C6 & Estrategias de ludificación \\
\hline
\end{tabular}

Fuente: Elaboración propia a partir del texto de (Sandí y Sanz, 2018a)

Seguido, se ejecuta una descripción de los criterios de análisis con el propósito de brindarle semántica a cada uno y posteriormente, hacer más fácil su comprensión.

- C1 País de origen del juego e idiomas que soporta - hace referencia al país donde fue desarrollado el juego y los posibles idiomas en los cuales se podría encontrar disponible.

- C2 Intensión pedagógica - hace referencia al objetivo principal que caracteriza al juego (entrenamiento, capacitación, desarrollo y/o adquisición de competencias, entre otros).

- C3 Guías/ayudas disponibles - se refiere a los diferentes tipos de ayuda que puede brindar el juego a los usuarios (manuales, tutoriales, guías de apoyo, preguntas frecuentes, sugerencias, entre otros).

- C4 Destinatarios del juego - se refiere a que si el juego desarrolla una descripción del público para el cual está orientado, es decir, si indican posibles edades o alguna población en específico.

- C5 Resultados de utilización - hace referencia a la existencia de resultados académicos y científicos que respalden los hallazgos obtenidos a partir de la implementación del juego.

- C6 Estrategias de ludificación - el juego describir la metodología de ludificación que utiliza (medallas, ranking, insignia, puntajes, premiación, entre otros). Aspecto relevante a considerar, debido a que los juegos serios se caracterizan por la intención pedagógica y, por incorporar estrategias lúdicas.

Posteriormente, ya con los criterios (C) definidos, se describe cada uno de los juegos elegidos.

\subsection{Juegos serios y su respectivo análisis a la luz de los criterios definidos}

La Tabla 2, presenta la selección de juegos serios para el estudio, los cuales han sido categorizados según las dimensiones expuestas por el Ministerio de Educación de Chile (2006), tales como: dimensión pedagógica, técnica, social-ética-legal, gestión y desarrollo profesional.

TABLA 2

Juegos serios elegidos

\begin{tabular}{lll}
\hline Código & Nombre del juego serio & Dimensión \\
\hline JSEx1 & Tamagocours & Pedagógica \\
\hline JSEx2 & NoviCraft & \\
\hline JSEx3 & Spirits of Spring & Social, Ética y Legal \\
\hline JSEx4 & Frecuency 1550 & Técnica \\
\hline JSEx5 & Cisco Packet Tracer & \\
\hline JSEx6 & GSD Sim & Gestión \\
\hline JSEx7 & RollerCoaster Tycoon Touch & Desarrollo \\
\hline JSEx8 & AstroCódigo & Profesional \\
\hline JSEx9 & RITA &
\end{tabular}

Fuente: Elaboración propia a partir del texto de (Sandí y Sanz, 2018a) 
Según el objetivo indicado en cada juego, así se le clasificará según dimensiones expuestas por el Ministerio de Educación de Chile (2006). En este sentido, al juego estar relacionado con las TIC y pedagogía, clasifica dentro de la Dimensión Pedagógica. O bien, el objetivo caracterizante está relacionado con los derechos del uso de las TIC, puede clasificarse dentro de la Dimensión Social, Ética y Legal. Luego, cuando la característica principal del juego está vinculada con la formación de competencias en relación al aprendizaje de hardware y software, clasifica dentro de la Dimensión Técnica. Seguido, cuando la característica principal del juego corresponde con la formación de competencias para potenciar el quehacer administrativo del profesorado y de la unidad académica, clasificar dentro de la Dimensión de Gestión. Posteriormente, cuando la característica principal del juego está relacionada con potenciar competencias para el intercambio de experiencias que permitan el mejoramiento de los procesos formativos, se les clasificará en la Dimensión de Desarrollo Profesional. Podría suceder que, los objetivos caracterizantes se puedas asociar a más de una dimensión. No obstante, para efectos de esta investigación, se le ubicará específicamente en una sola dimensión específica. A continuación, se describe el objetivo de los juegos elegidos, posteriormente, se desarrollará el análisis según dimensiones y criterios definidos.

\section{A) Dimensión - Pedagógica}

En esta sección, se presentan los juegos serios Tamagocours y NoviCraft los cuales posibilitan al personal docente la adquisición de competencias que les permite utilizar, aplicar e integrar las TIC de una forma más novedosa en su quehacer docente.

JSEx1 Tamagocours

Tamagocours es un juego serio de tipo aplicación web el cual puede ser accedido de forma gratuita, de código fuente cerrado (no se pueden efectuar modificaciones al código fuente), se caracteriza por ser multi-tenant (soportar múltiples usuarios, en este caso jugadores). Fue desarrollado para el entrenamiento del profesorado en competencias digitales e internet, para que les permitan utilizar e integrar las TIC en los procesos formativos (Sánchez y Emin, 2014). Otro aspecto importante a considerar de Tamagocours, consiste en la posibilidad de formar competencias relacionadas con los derechos de autor, específicamente, al explotarse ciertos recursos tecnológicos, por ello, se le podría clasificar también en la dimensión ética y legal (Sánchez, Emin y Mandran, 2015).

JSEx2 NoviCraft

NoviCraft es un juego serio 3D de tipo aplicación de escritorio el cual puede ser accedido mediante el pago de suscripción, de código fuente cerrado y se caracteriza por ser multi-tenant (Holloway, 2009). Su objetivo caracterizante radica mediante el adiestramiento potenciar la adquisición de habilidades pedagógicas del profesorado, mediante la incorporación, utilización y aplicación de TIC en procesos formativos, donde el profesorado implementa el trabajo en equipo, la gestión y el liderazgo. Se ha evidenciado aumento en la motivación e interés por parte de los participantes al utilizar NoviCraft (Juzeleniene, Mikelioniene, Escudeiro y Vaz de Carvalho, 2014).

El reto consiste en desarrollar en un contexto cambiante, donde se hallarán jugadores con distintos tipos de actitudes y habilidades, los cuales se deben unir para definir estrategias para ejecutar acciones con el objetivo de alcanzar el cumplimiento de las metas propuestas en el juego, mediante el trabajo y la colaboración (Romero, Usart y Ott, 2015).

En resumen, los juegos (Tamagocours y NoviCraft) han sido utilizados para potenciar estrategias de negociación, adquisición de competencias pedagógicas, favorecer la metacognición y competencias relacionadas con los derechos de autor de materiales educativos. Acá es importante destacar su característica Multi-Tenant, la cual podría permitir la generación de habilidades de colaboración, las cuales permiten mejorar los procesos formativos.

B) Dimensión - Social, Ética Y Legal 
Los juegos Spirits of Spring y Frecuency 1550, posibilitan que el profesorado y estudiantado conozcan, adquieran, se apropien y difundan conocimientos vinculados con aspectos sociales, éticos y legales de las tecnologías digitales.

JSEx3 Spirits of Spring

Spirits of Spring es un juego serio emotivo 3D para dispositivos móviles el cual puede ser accedido mediante un pago mínimo de suscripción, de código fuente cerrado y se caracteriza por ser singletenant (soporta únicamente un usuario o jugador, es decir, se juega de forma individual). Su objetivo caracterizante se vincula con la concientización del profesorado y estudiantado en aspectos socialeséticos-legales vinculados a las tecnologías digitales, específicamente, prevenir el uso de las TIC para potenciar el bullying. El personaje protagonista tendrá que enfrentar a intimidación desde el punto de vista del matón, la víctima y el espectador (OmniumGames, 2014).

JSEx4 Frecuency 1550

Frecuency 1550 es un juego serio para dispositivos móviles (App 7scenes), el cual puede ser accedido mediante el pago de suscripción, de código fuente cerrado y se caracteriza por ser multi-tenant. Su objetivo caracterizante se vincula con la adquisición de conocimientos relacionados con los aspectos sociales y culturales de la historia medieval (Apezteguía, Rapetti, Gordillo y Challiol, 2014).

Como actividad, propone la búsqueda de la famosa Sagrada Hostia, para ello, los jugadores/as deben recorrer libremente la ciudad mientras permanecen interconectados para contestar preguntas basadas en asignaciones de localización mediante la utilización de GPS de sus dispositivos (WaagSociety, 2019).

En resumen, los juegos presentados favorecen la obtención de resultados relacionados con la adquisición de habilidades sociales-éticas-legales vinculadas con las TIC. Igualmente, se vislumbra su potencial para activar la metacognición (reflexión en torno a las estrategias y emociones para alcanzar la superación de los retos y desafíos del juego) y la memoria. Se evidencia que ambos juegos son coherentes en relación a sus objetivos propuestos (mensaje, diseño gráfico y multimedia). Particularmente, se destaca que ambos juegos permiten la generación y/o formación de competencias digitales a través del uso de dispositivos tecnologías móviles.

\section{C) Dimensión - Técnica}

En esta sección se describen dos juegos Cisco Packet Tracer y GSD Sim, los cuales brindan a la población docente y estudiantil la posibilidad de la formación de competencias digitales relacionadas con el empleo de las TIC, proliferación de nuevos conocimientos y destrezas asociados a hardware y software, los cuales podrían serles de utilidad en su práctica académica y profesional.

\section{JSEx5 Cisco Packet Tracer}

Cisco Packet Tracer es un juego serio de simulación, de aplicación tipo mixta (móvil y escritorio), el cual puede ser accedido de forma gratuita, de código fuente cerrado y se caracteriza por ser multi-tenant (Janitor, Jakab y Kniewald, 2010). Fue desarrollado por Cisco Systems, inicialmente se consideró un software de simulación educativo utilizado como medio de apoyo en Cisco Networking Academy, el cual está dirigido a todo el público (Cabarkapa, 2015).

Posteriormente, en Rumania un grupo de investigadores desarrollan un módulo llamado PTActivity el cual permite la integración de las potencialidades de Cisco Packet Tracer con Moodle, permitiendo la crear actividades y estrategias de aprendizaje tipo e-learning, por ello, brinda la posibilidad de mejorar el proceso formativo en redes (Janitor et al., 2010; Petcu, Iancu, Peculea, Dadarlat y Cebuc, 2013). La integración del módulo PTActivity permite considerar a Cisco Packet Tracer como un juego serio.

En sí, el objetivo caracterizante de Cisco Packet Tracer se vincula con la formación de habilidades digitales, trabajo colaborativo y las redes de datos a través del entrenamiento y la experimentación brindada mediante la simulación y la ludificación. 
Cisco Packet Tracer permite al profesorado crear diferentes tipos de estrategias didácticas complejas mediante la simulación y visualización; permite al jugador decidir el tipo de visualización de su preferencia (en tiempo real o simulado), es decir, se puede crear topologías de red y simular la ejecución de un proceso dentro de la simulación.

JSEx6 GSD Sim (GSD, Global Software Development).

GSD Sim es un juego serio de simulación, de aplicación tipo web, el cual puede ser accedido de forma gratuita y requiere de registro de usuarios, de código fuente cerrado y se caracteriza por ser multi-tenant. Disponible en: http://proisis.lero.ie/gsd-sim/, su objetivo caracterizante se vincula con la potencialización de las habilidades en la implementación de software global. El reto del juego consiste en que la persona jugadora debe vencer los problemas e inconvenientes actuales del desarrollo de software (cambios a nivel de requerimientos por parte de los usuarios, falta de claridad del problema por resolver, zonas horarias, barreras geográficas, idiomáticas, culturales, entre otras), en fin, a todas esas barreras en el desarrollo de software se les menciona como distancia global (Noll, Beecham y Richardson, 2010).

En suma, ambos juegos (Cisco Packet Tracery GSD Sim) corresponden al entrenamiento, los cuales han permitido desarrollar habilidades para la configuración de dispositivos y sistemas globales, donde se deben tomar ciertas decisiones vinculadas con el manejo de los tiempos y costes. Estos juegos podrían asociarse con la dimensión de Gestión.

En síntesis, a nivel de educación superior, ambo juegos evidencian que podrían ser utilizados para formar diferentes tipos de competencias en el profesorado, las cuales van desde la generación de destrezas, adquisición de habilidades digitales y posibilidades de brindar feedback.

D) Dimensión - Gestión

En esta sección, se presenta el juego serio RollerCoaster Tycoon el cual permite potenciar competencias de gestión vinculadas con el pensamiento estratégico, toma de decisiones y habilidades de planificación. Este juego podría ser útil para el profesorado y para el estudiantado, cuyas habilidades a adquirir se pueden utilizar como medio de apoyo en el mejoramiento continuo de la institución educativa.

\section{JSEx7 RollerCoaster Tycoon}

RollerCoaster Tycoon es juego serio para dispositivos móviles, el cual puede ser accedido de forma gratuita y requiere de registro de usuarios, de código fuente cerrado y se caracteriza por ser single-tenant. Su objetivo caracterizante se vincula con la formación de competencias de gestión, específicamente, las vinculadas con formación del pensamiento, toma de decisiones y planificación estratégica.

El desafío del juego consiste en la construcción de un parque temático, mediante el cual el jugador tendrá que resolver una serie de desafíos que le permitirán desarrollar competencias de gestión, planeamiento estratégico, manejo de inversiones, detección de necesidades y requerimientos (Romero y Turpo, 2012; Foster, 2011).

En síntesis, RollerCoaster Tycoon favorece la adquisición de habilidades y destrezas de gestión y planificación estratégica, las cuales son requeridas en el profesorado para mejorar los procesos formativos a nivel de educación superior, lo cual está acorde a lo indicado por el Ministerio de Educación de Chile.

\section{E) Dimensión - Desarrollo Profesional}

AstroCódigo es un juego serio para PC, el cual puede ser accedido de forma gratuita y requiere de registro de usuarios, de código fuente cerrado y se caracteriza por ser single-tenant. Disponible en: htt p://www.astrocodigo.com, su objetivo caracterizante se vincula con el acercamiento del jugador a los conceptos básicos de la programación. Ofrece la posibilidad de formar competencias y habilidades digitales vinculadas con la indagación de conceptos relacionados con hardware y software, a la 
resolución de problemas a través de la estructuración del pensamiento (de manera tal que se potencia la organización y resolución de dificultades de forma organizada) a través del uso de la algoritmia y lo lúdico. El reto del juego consiste en la resolución de escenarios de manera algorítmica y lúdica. De esta manera se ayuda a estructurar el pensamiento, a organizarlo y a resolver problemas de manera estructurada (Bione, Miceli, Sanz y Artola, 2017).

En síntesis, AstroCódigo le permitiría al profesorado diseñar actividades de formación y capacitación innovadoras vinculadas con la programación y la potencialización del pensamiento computacional.

\section{JSEx9 Rita}

RITA es un juego serio para PC, el cual puede ser accedido de forma gratuita y requiere de registro de usuarios, de código fuente abierto y se caracteriza por ser single-tenant. Su objetivo caracterizante se vincula con la formación de competencias tecnológicas relacionadas con la programación, específicamente, orientado a potenciar la lógica de la programación y la algoritmia en el estudiantado y profesorado a nivel de educación secundaria. Igualmente, RITA posibilita la formación de habilidades en la programación, específicamente la relacionada con objetos, además, el lenguaje Java (Aybar, Queiruga y Banchoff, 2012).

El reto del juego consiste en la programación de robots para ir al campo de batalla. Para la configuración de los robots se utiliza la programación de algoritmos a través de la técnica de la programación por bloques (Queiruga et al., 2016).

En suma, ambos juegos (AstroCódigo y RITA) favorecen la adquisición de competencias necesarias en la programación y la potencialización del pensamiento computacional mediante el desarrollo de estrategias lúdicas y sociales creativas.

La Tabla 3, resume las características de los juegos serios según criterios de análisis. 
TABLA 3

Resumen características de los juegos serios según criterios de análisis

\begin{tabular}{|c|c|c|c|c|c|c|c|}
\hline \multirow{2}{*}{ Dimensión } & \multirow{2}{*}{ Juegos serios } & \multicolumn{6}{|l|}{ Criterios de análisis } \\
\hline & & $\mathrm{C1}$ & $\mathrm{C} 2$ & $\mathrm{C3}$ & $\mathrm{C4}$ & C5 & C6 \\
\hline \multirow[b]{2}{*}{$\begin{array}{l}\text { Pedagógica } \\
\text { (Docencia) }\end{array}$} & Tamagocours & Francia Francés & $\begin{array}{l}\text { Entrenamiento; } \\
\text { competencias } \\
\text { digitales }\end{array}$ & $\begin{array}{l}\text { Tutoriales } \\
\text { y manuales }\end{array}$ & Docentes & $\begin{array}{l}\text { Activa la } \\
\text { metacognición } \\
\text { y colaboración }\end{array}$ & $\begin{array}{l}\text { Puntos y } \\
\text { certificados }\end{array}$ \\
\hline & NoviCraft & $\begin{array}{l}\text { Finl andia Finés e } \\
\text { inglés }\end{array}$ & $\begin{array}{l}\text { Entrenamiento; } \\
\text { competencias } \\
\text { pedagógicas }\end{array}$ & $\begin{array}{l}\text { Soporte, } \\
\text { tutoriales y } \\
\text { manuales }\end{array}$ & $\begin{array}{l}\text { Docentes y } \\
\text { estudiantes }\end{array}$ & $\begin{array}{l}\text { Exitoso, } \\
\text { entretenido y } \\
\text { muy } \\
\text { motivador }\end{array}$ & Puntos \\
\hline \multirow[t]{2}{*}{$\begin{array}{l}\text { Social, Ética } \\
\text { y Legal }\end{array}$} & $\begin{array}{l}\text { Spirits of } \\
\text { Spring }\end{array}$ & $\begin{array}{l}\text { Canadá Francés, } \\
\text { inglés, español, } \\
\text { italiano alemán, } \\
\text { portugués }\end{array}$ & $\begin{array}{l}\text { Concientización: } \\
\text { aspectos } \\
\text { sociales, éticos } \\
\text { y legales del } \\
\text { uso de las TIC }\end{array}$ & $\begin{array}{l}\text { Soporte e } \\
\text { información } \\
\text { en redes } \\
\text { sociales }\end{array}$ & Estudiantes & Entretenido & Puntos \\
\hline & $\begin{array}{l}\text { Frecuency } \\
1550\end{array}$ & $\begin{array}{l}\text { Holanda } \\
\text { Neerlandés e inglés }\end{array}$ & $\begin{array}{l}\text { Aprendizaje: } \\
\text { competencias } \\
\text { digitales }\end{array}$ & $\begin{array}{l}\text { Tutoriales, } \\
\text { videos y } \\
\text { manuales }\end{array}$ & Estudiantes & $\begin{array}{l}\text { Entretenido, } \\
\text { metacognición } \\
\text { y la memoria }\end{array}$ & Puntos \\
\hline \multirow[t]{2}{*}{ Técnica } & $\begin{array}{l}\text { Cisco Packet } \\
\text { Tracer }\end{array}$ & $\begin{array}{l}\text { EEUU Inglés, } \\
\text { español, alemán, } \\
\text { francés, ruso y } \\
\text { portugués }\end{array}$ & $\begin{array}{l}\text { Entrenamiento; } \\
\text { competencias } \\
\text { digitales, } \\
\text { trabajo } \\
\text { colaborativo }\end{array}$ & $\begin{array}{l}\text { Tutoriales, } \\
\text { videos y } \\
\text { manuales }\end{array}$ & $\begin{array}{l}\text { Todo } \\
\text { público }\end{array}$ & $\begin{array}{l}\text { Exitoso, } \\
\text { entretenido y } \\
\text { muy } \\
\text { motivador }\end{array}$ & Puntos y feedback \\
\hline & GSD Sim & Irlanda Inglés & $\begin{array}{l}\text { Entrenamiento; } \\
\text { competencias } \\
\text { digitales }\end{array}$ & Tutoriales & Estudiantes & $\begin{array}{l}\text { Entretenido, } \\
\text { integración de } \\
\text { conocimientos }\end{array}$ & $\begin{array}{l}\text { Puntos y dinero } \\
\text { virtual }\end{array}$ \\
\hline Gestión & $\begin{array}{l}\text { RollerCoaster } \\
\text { Tycoon } \\
\text { Touch }\end{array}$ & $\begin{array}{l}\text { Canadá Inglés, } \\
\text { francés, italiano, } \\
\text { alemán, español, } \\
\text { sueco, ruso, } \\
\text { holandés, } \\
\text { portugués. }\end{array}$ & $\begin{array}{l}\text { Entrenamiento; } \\
\text { Competencias } \\
\text { digitales, } \\
\text { pensamiento } \\
\text { estratégico }\end{array}$ & $\begin{array}{l}\text { Preguntas } \\
\text { frecuentes, } \\
\text { tutoriales y } \\
\text { soporte } \\
\text { técnico }\end{array}$ & $\begin{array}{l}\text { Todo } \\
\text { público }\end{array}$ & $\begin{array}{l}\text { Exitoso, } \\
\text { entretenido y } \\
\text { muy } \\
\text { motivador }\end{array}$ & $\begin{array}{l}\text { Monedas, bonos de } \\
\text { cartas, desbloqueo } \\
\text { de niveles y } \\
\text { feediback }\end{array}$ \\
\hline \multirow[t]{2}{*}{$\begin{array}{l}\text { Desarrollo } \\
\text { Profesional }\end{array}$} & AstroCódigo & $\begin{array}{l}\text { Argentina Español } \\
\text { e inglés }\end{array}$ & $\begin{array}{l}\text { Aprendizaje; } \\
\text { competencias } \\
\text { digitales y } \\
\text { desarrollo del } \\
\text { pensamiento } \\
\text { computacional }\end{array}$ & $\begin{array}{l}\text { Tutoriales } \\
\text { y guias }\end{array}$ & $\begin{array}{l}\text { Docentes } \\
\mathrm{y} \\
\text { Estudiantes }\end{array}$ & $\begin{array}{l}\text { Motivador, } \\
\text { activa } \\
\text { metacognición, }\end{array}$ & $\begin{array}{l}\text { Insignias, puntos, } \\
\text { feedback y ranking }\end{array}$ \\
\hline & RITA & Argentina Español & $\begin{array}{l}\text { Aprendizaje; } \\
\text { competencias } \\
\text { digitales }\end{array}$ & $\begin{array}{l}\text { Bloques } \\
\text { con ayuda }\end{array}$ & $\begin{array}{l}\text { Docentes y } \\
\text { estudiantes }\end{array}$ & $\begin{array}{l}\text { Muy } \\
\text { motivador. } \\
\text { Lúdico y social }\end{array}$ & Puntos, feediback \\
\hline
\end{tabular}

Fuente: Elaboración propia, 2019.

En la próxima sección, se analiza en detalle los juegos serios descritos según criterios definidos y resumidos en la Tabla 3 (esta sección permite brindar respuesta a la RQ6).

\subsection{Análisis general de los juegos serios elegidos}

Acorde a los hallazgos obtenidos en el análisis aplicado de forma individual a cada uno de los juegos serios descritos, se identificaron resultados generales vinculados directamente con cada uno de los criterios de análisis definidos para esta investigación.

Este análisis general tiene el propósito de exponer una serie de características y fortalezas vinculadas a los juegos serios elegidos. Ahora bien, se debe tener en cuenta que la muestra es pequeña, por ello, no es posible establecer una tendencia o brindar conclusiones contundentes, sin embargo, permite dar a conocer las características globales de os juegos seleccionados. La introducción de porcentajes para las descripciones de los criterios se orienta en este sentido.

C1 País de origen del juego e idiomas que soporta- Más de la mitad de los juegos serios estudiados son de américa, se registran juegos de Canadá (2), Estados Unidos (1) y Argentina (2), para un total del 55.56\%. El otro 44.44\% corresponde a países europeos, quienes registran un juego por cada país; Irlanda, Francia, Holanda y Finlandia. Un alto porcentaje (66.67\%) de los juegos serios analizados (6 de 9) ofrecen su 
disponibilidad entre dos a nueve idiomas, por ejemplo, RollerCoaster Tycoon Touch se encuentra disponible en 9 idiomas. Luego, Spirits of Spring registra 6 leguajes distintos al igual que Cisco Packet Tracer. Seguido, están los que soportan dos idiomas: AstroCódigo (español e inglés), NoviCraft (Finés e inglés) y Frecuency 1550 (neerlandés e inglés). El restante $33.33 \%$ de los juegos ofrece su disponibilidad en un solo idioma (3 de 9); Tamagocours (francés) y GSD SIM (inglés) y RITA (español). Lo anterior, evidencia que se ha considerado y tomado en cuenta durante su desarrollo las barreras lingüísticas, lo cual se evidencia como una fortaleza de los juegos analizados, debido a que las aplicaciones en múltiples idiomas permiten ser más comprendidas y utilizadas por diferentes usuarios en otros países e incluso continentes.

C2 Intensión pedagógica (objetivo caracterizante) - En la mayor parte de los juegos estudiados (66.67\%) el objetivo caracterizante está vinculado directamente con el entrenamiento en habilidades y destrezas para la adquisición de competencias digitales. Luego, el 33.33\% de los juegos serios, tienen como objetivo principal y caracterizante el aprendizaje, además, posibilitan la formación o adquisición de competencias digitales. Entre las competencias digitales identificadas a desarrollar por parte de los juegos serios analizados, van desde la dimensión docente, implicación legal de las tecnologías, al trabajo colaborativo, desarrollo del pensamiento estratégico, formación en habilidades en gestión y planificación con tecnologías. Lo anterior, evidencia que aún y cuando los juegos serios analizados no fueron pensados para potenciar directamente el desarrollo o adquisición de competencias digitales, sin embargo, logran alcanzar dicho propósito. Lo cual es visto como una fortaleza, debido a que se indiferentemente de su intención pedagógica inicial, algunos de los juegos se podrían adaptar acorde a los requerimientos particulares de cada institución educativa desee implementarlos.

C3 Guías/ayudas disponibles - En su totalidad, los juegos estudiados ofrecen diferentes tipos de ayuda, estas van desde manuales, videotutoriales, preguntas frecuentes, soporte técnico, guías pedagógicas, entre otras. Fortaleza destacable, pue la variedad de tipos de ayuda ofrecidos, permitirían que usuarios con diferentes capacidades de aprendizaje, puedan comprender con mayor facilidad el objetivo y las actividades por ejecutar en el juego.

C4 Destinatarios del juego - En su totalidad, los juegos estudiados potencian la adquisición de competencias digitales en la población docente y estudiantil. No obstante, Tamagocours es utilizado exclusivamente para potenciar competencias digitales en el profesorado. Lo anterior, se toma como una fortaleza, debido a que, si bien, existen juegos serios orientados para ser mayormente utilizados con la población estudiantil, resultan interesantes y efectivos al aplicarse con docentes.

C5 Resultados de utilización - En su totalidad, los juegos estudiados evidenciaron resultados positivos en términos de ser calificados por parte de las personas jugadoras como motivadores, entretenidos, exitosos y, particularmente, caracterizados por permitir activar la metacognición y, potenciar el pensamiento crítico.

C6 Estrategias de ludificación - En su totalidad, los juegos estudiados reflejaron la utilización de alguna metodología o estrategia de ludificación, algunos juegos presentaron mayor cantidad de estrategias y variedad de estas, sin embargo, todos utilizan al menos una estrategia. Entre las principales estrategias están; ranking, feedback, asignación de puntos, insignias, dinero virtual, desbloqueo de niveles, entre otros. Lo anterior, permite fortalecer el proceso formativo, debido a que, al existir una variedad de estrategias de ludificación, es más probable un aumento en la motivación de los usuarios hacia las actividades por desarrollar, con ello, se lograría alcanzar la intensión pedagógica del juego.

Adicionalmente, se ha identificado a través de la revisión y descripción de los juegos serios analizados, otras fortalezas de los juegos serios importantes a resaltar:

$\checkmark$ Se identificó en la mayor parte de los juegos serios analizados, que su acceso a la aplicación es gratuito por parte del usuario, lo cual es una fortaleza, ya que les permitiría alcanzar un mayor impacto en relación a cobertura y acceso de usuarios. Particularidad importante para ser tomada en cuenta al momento que se requiera diseñar e implementar un juego serio.

$\checkmark$ Se constató que los desarrolladores de los juegos serios analizados, en su mayoría, orientaron sus aplicaciones para ser ejecutadas desde dispositivos móviles. Aspecto que se convierte en una fortaleza, debido 
a que en la actualidad el acceso y uso de las tecnologías móviles es más frecuente en los usuarios (Cruz y Sandí, 2017; Sandí y Cruz, 2018).

$\checkmark$ La característica multi-tenant presente mayoritariamente en los juegos serios analizados, podría indicar que existe la posibilidad de utilizar estos juegos para desarrollar actividades grupales con el profesorado en tiempo real para la construcción de experiencias académicas conjuntas y colaborativas (análisis de situaciones y toma de decisiones en forma colaborativa), las cuales podrían impulsar una mejora significativa en los procesos formativos.

En el próximo apartado, se presenta las principales conclusiones arribadas a partir de la investigación efectuada, así como las posibles líneas de trabajo futuro.

\section{CONCLUSIONES Y LÍNEA DE TRABAJO FUTURo}

La investigación elaborada permitió indagar, recopilar y describir una serie de juegos serios, los cuales fueron analizados acorde a las fortalezas que brindan en relación a la adquisición de competencias dígales en la formación del personal académico.

A partir del estudio desarrollado, se reconocen en los juegos serios analizados, algunas potencialidades que favorecen la adquisición de competencias digitales (ej. aplicaciones multilenguaje, orientación al acceso gratuito, múltiples tipos de ayuda disponibles, variedad de estrategias de ludificación, entre otras), fortalezas que pueden servir de guía u orientación a las instituciones educativas a nivel general y, en particular, a las de educación superior, que anhelen utilizar e integrar los juegos serios para la formación y/o adquisición de competencias digitales por parte del profesorado.

Se observa que el uso de los juegos serios se ha enfocado mayoritariamente para la interacción con la población estudiantil a nivel de educación secundaria y educación universitaria. A nivel del profesorado, se evidencia que existe un reducido manejo de juegos serios en la formación y capacitación docente. Sin embargo, se identifican fortalezas o posibilidades que brindan los juegos serios al personal académico para fortalecer los procesos formativos (ej. formación de diferentes habilidades en competencias digitales, de gestión, pensamiento y planificación estratégica, trabajo colaborativo, entre otras).

Acorde a los resultados de utilización de los juegos serios, estos han sido catalogados como recursos educativos innovadores, creativos y divertidos, los cuales permiten al profesorado un aprendizaje crítico y flexible en diferentes áreas del conocimiento, en particular, la formación de competencias digitales y potenciar el pensamiento computacional. Se resalta la motivación y una actitud favorable por parte de la población usuaria hacia las tecnologías digitales, específicamente, los juegos serios.

Como trabajo futuro se ahondará en la elaboración de una propuesta para la aplicación de uno los juegos serios analizados (AstroCódigo) para potenciar la adquisición y/o formación de competencias digitales por parte del personal docente a nivel de educación superior. Lo anterior, debido a que se ha constatado que el juego citado permite: a) la formación de competencias digitales, $b$ ) indagación de conceptos como el de hardware y software, $c$ ) resolución de problemas de forma sistematizada, lo cual permitirá fortalecer el pensamiento estructurado requerido durante la planificación del uso de las tecnologías digitales (Sandí y Sanz, 2018a). Además, se requiere brindar continuación a esta línea de investigación para trabajar en el diseño y desarrollo de otros juegos serios concretamente orientados a la formación de competencias digitales en el profesorado a nivel de educación superior, según requerimientos o necesidades específicas de las instituciones educativas.

\section{RefEREnCias}

Abt, C. C. (1970). Serious games. New York: Viking Press. 
Angelini, M. L., García, A. y Martínez, N. (2017). Estudio de correlación entre la simulación telemática y las destrezas lingüísticas en inglés. REDIE. Revista Electrónica de Investigación Educativa, 19(1), 141-156. doi: https://doi. org/10.24320/redie.2017.19.1.1100

Apezteguía, M., Rapetti, D. E., Gordillo, S. E. y Challiol, C. (2014).Juego educativo móvil colaborativo. (Tesis de grado). Universidad Nacional de La Plata (UNLP), Buenos Aires, Argentina. Recuperado de http://hdl.handle.net/1 $0915 / 47078$

Aybar, V. del C., Queiruga, C. y Banchoff, C. (2012). Aplicaciones complementarias a ROBOCODE que faciliten el aprendizaje de programación en escuelas secundarias. (Tesis de grado). Universidad Nacional de La Plata (UNLP), Buenos Aires, Argentina. Recuperado de http://hdl.handle.net/10915/47050

Bione, J., Miceli, P., Sanz, C. V. y Artola, V. (2017). AstroCódigo. Un juego serio para la introducción de jóvenes en los conceptos básicos de la programación. (Tesis de grado). Universidad Nacional de La Plata (UNLP), Buenos Aires, Argentina. Recuperado de http://hdl.handle.net/10915/61204

Cabarkapa, D. (2015). Application of Cisco Packet Tracer 6.2 in teaching of advanced computer networks. doi: https:// doi.org/10.13140/RG.2.1.4881.6802

Charsky, D. (2010). From edutainment to serious games: A change in the use of game characteristics. Games and Culture, 5(2), 177-198. doi: https://doi.org/10.1177/1555412009354727

Choi, M., Cristol, D. y Gimbert, B. (2018). Teachers as digital citizens: The influence of individual backgrounds, internet use and psychological characteristics on teachers' levels of digital citizenship. Computers \& Education, 121, 143-161. doi; https://doi.org/10.1016/j.compedu.2018.03.005

Claro, M., Salinas, A., Cabello, T., San Martín, E., Preiss, D. D., Valenzuela, S. y Jara, I. (2018). Teaching in a digital environment (TIDE): Defining and measuring teachers' capacity to develop students' digital information and communication skills. Computers \& Education, 121, 162-174. doi: https://doi.org/10.1016/j.compedu.2018.0 3.001

Connolly, T. M., Boyle, E. A., MacArthur, E., Hainey, T. y Boyle, J. M. (2012). A systematic literature review of empirical evidence on computer games and serious games. Computers \& Education, 59(2), 661-686. doi: https ://doi.org/10.1016/j.compedu.2012.03.004

Cruz, E. D. C. (2018). Importancia del manejo de competencias tecnológicas en las prácticas docentes de la Universidad Nacional Experimental de la Seguridad (UNES). Revista Educación, 43(1), 1-23. doi: https:// doi.org/10.15517/revedu.v43i1.27120

Cruz, M. A. y Bazán, P. A. (2018). Understanding the internet of nano things: Overview, trends, and challenges. eCiencias de la Información, 9(1), 1-30. doi: https://doi.org/10.15517/eci.v1i1.33807

Cruz, M. A. y Sandí, J. C. (2017). Sistemas y tecnologías que facilitan el posicionamiento indoor. Revista Pensamiento Actual, 17(29), 132-144. Recuperado de https://revistas.ucr.ac.cr/index.php/pensamiento-actual/article/view 131585

Cruz, M. A., Sandí, J. C. y Víquez, I. G. (2017). Diseño de situaciones educativas innovadoras como estrategia didáctica para fortalecer el proceso de enseñanza-aprendizaje. Revista Didasc@lia: Didáctica y Educación, 8(8), 99-116. Recuperado de http://hdl.handle.net/10915/61270

Dávila, A. (2014). Simulación en Educación Médica. Investigación en Educación Médica, 3(10), 100-105. doi: https: //doi.org/10.1016/S2007-5057(14)72733-4

Foster, A. N. (2011). The process of learning in a simulation strategy game: Disciplinary knowledge construction. Journal of Educational Computing Research, 45(1), 1-27. doi: https://doi.org/10.2190/EC.45.1.a

Guenaga, M., Eguíluz, A., Rayón, A. y Quevedo, E. (2015). Un juego serio para desarrollar y evaluar la competencia de trabajo en equipo. Revista Iberoamericana de Informática Educativa, (21), 3-11. Recuperado de http://dialn et.unirioja.es/servlet/articulo?codigo $=5113264$

Holloway, D. (2009). NoviCraft: virtual world team building. The Creative Shed. Recuperado de https://bit.ly/2Lk $\mathrm{pC} 8 \mathrm{q}$ 
Juan Carlos Sandí Delgado, et al. Juegos Serios para potenciar la adQuisición de competencias digi...

Janitor, J., Jakab, F. y Kniewald, K. (marzo, 2010). Visual Learning Tools for Teaching/Learning Computer Networks: Cisco Networking Academy and Packet Tracer. Trabajo presentado en la Sixth International Conference on Networking and Services, Cancún, México. https://doi.org/10.1109/ICNS.2010.55

Juzeleniene, S., Mikelioniene, J., Escudeiro, P. y Vaz de Carvalho, C. (2014). GABALL project: Serious games based language learning. Procedia - Social and Behavioral Sciences, 136, 350-354. doi: https://doi.org/10.1016/j.sbsp ro.2014.05.340

Kitchenham, B., Brereton, O. P., Budgen, D., Turner, M., Bailey, J. y Linkman, S. (2009). Systematic literature reviews in software engineering - A systematic literature review. Information and Software Technology, 51(1), 7-15. doi: https://doi.org/10.1016/j.infsof.2008.09.009

Lee, H. y Cho, C.-H. (2017). An application of brand personality to advergames: The effect of company attributes on advergame personality. Computers in Human Behavior, 69, 235-245. doi: https://doi.org/10.1016/j.chb.2 016.12 .035

López, C. (2016). El videojuego como herramienta educativa. Posibilidades y problemáticas acerca de los serious games. Apertura. Revista de Innovación Educativa, 8(1), 136-151. Recuperado de https://dialnet.unirioja.es/servlet/ar ticulo?codigo $=5547036$

Ma, M. y Oikonomou, A. (2017). Serious games and edutainment applications. United Kingdom: Springer, Cham. h ttps://doi.org/10.1007/978-3-319-51645-5

Martínez, L. J. (2016). Cómo buscar y usar información cientifica: Guia para estudiantes universitarios 2016. Santander, España: Universidad de Cantabri. Recuperado de http://eprints.rclis.org/29934/7/Como_buscar_usar_infor macion_2016.pdf

Michael, D. y Chen, S. L. (2006). Serious games: games that educate, train and inform. Boston, MA, USA: Thomson Course Technology.

Ministerio de Educación de Chile. (2006). Estándares en tecnología de la información y la comunicación para la formación inicial docente. Santiago: Ministerio de Educación de Chile.

Noll, J., Beecham, S. y Richardson, I. (2010). Global software development and collaboration: barriers and solutions. ACM Inroads, 1(3), 66-78. doi: https://doi.org/10.1145/1835428.1835445

OmniumGames. (2014, octubre). Spirits of Spring: Una emocionante aventura contra el bullying. The Game Journal. Recuperado de https://bit.ly/108ydSf

Padilla, N., Gutiérrez, F. L. y Medina, N. (2011). Metodología para el diseño de videojuegos educativos sobre una arquitectura para el análisis del aprendizaje colaborativo. (Tesis doctoral). Universidad de Granada, Granada, España. Recuperado de http://hdl.handle.net/10481/19440

Petcu, D., Iancu, B., Peculea, A., Dadarlat, V. y Cebuc, E. (setiembre, 2013). Integrating Cisco Packet Tracer with Moodle platform: Support for teaching and automatic evaluation. Trabajo presentado en la RoEduNet International Conference 12th Edition: Networking in Education and Research, Iasi, Rumania. https://doi.org/ 10.1109/RoEduNet.2013.6714190

Prendes, M. P., Gutiérrez, I. y Martínez, F. (2018). Competencia digital: una necesidad del profesorado universitario en el siglo XXI. RED. Revista de Educación a Distancia, (56), 1-22. Recuperado de https://revistas.um.es/red/ article/view/321591

Queiruga, C., Fava, L., Banchoff, C., Aybar, V. del C., Kimura, I. M. y Brown, M. (2016). RITA: una herramienta didáctica-pedagógica innovadora en la escuela secundaria. Argentina: LINTI. Recuperado de https://bit.ly/2zw MfRx

Rodríguez, A.M., Raso, F. y Ruiz, J. (2019). Competencia digital, educación superior y formación del profesorado: Un estudio de meta-análisis en la web of science. Pixel-Bit. Revista de Medios y Educación, (54), 65-82. doi: https:/ /doi.org/10.12795/pixelbit.2019.i54.04

Roettl, J., Waiguny, M. y Terlutter, R. (2016). The persuasive power of advergames: A content analysis focusing on persuasive mechanisms in advergames. Australasian MarketingJournal, 24(4), 275-287. doi: https://doi.org/1 0.1016/j.ausmj.2016.10.001 
Romero, M. y Turpo, O. (2012). Serious games para el desarrollo de las competencias del siglo XXI. RED. Revista de Educación a Distancia, 34(1), 1-22. Recuperado de https://revistas.um.es/red/article/view/233511

Romero, M., Usart, M. y Ott, M. (2015). Can serious games contribute to developing and sustaining 21 st century skills? Games and Culture, 10(2), 148-177. doi: https://doi.org/10.1177/1555412014548919

Sánchez, É. y Emin, V. (octubre, 2014). Towards a model of play: An empirical study. Trabajo presentado en la European Conference on Games Based Learning, Reino Unido. Recuperado de https://search.proquest.com/do cview $/ 1674245678$ ?accountid $=15870$

Sánchez, É., Emin, V. y Mandran, N. (2015). Jeu-game, jeu-play, vers une modélisation du jeu. Une étude empirique à partir des traces numériques d'interaction du jeu Tamagocours. Sciences et Technologies de l'Information et de la Communication pour l'Éducation et la Formation, 22, 9-44. Recuperado de https://www.persee.fr/doc/stice 1764-7223_2015_num_22_1_1685

Sandí, J. C. y Bazán, P. A. (2019). Educational serious games as a service: Challenges and solutions. JCS ひ T. Journal of Computer Science and Technology, 19(01), 66-80. doi: https://doi.org/10.24215/16666038.19.e07

Sandí, J. C. y Cruz, M. A. (2016). Propuesta metodológica de enseñanza y aprendizaje para innovar la educación superior. Revista InterSedes, 17(36), 1-38. doi: https://doi.org/10.15517/isucr.v17i36.27100

Sandí, J. C. y Cruz, M. A. (2017a). La Simulación como recurso electrónico para potenciar las habilidades cognitivas del estudiantado. Revista InterSedes, 18(37), 1-31. doi: https://doi.org/10.15517/isucr.v18i37.28646

Sandí, J. C.y Cruz, M. A. (2017b). Repositorios institucionales digitales: Análisis comparativo entre Sedici (Argentina) y Kérwá (Costa Rica). e-Ciencias de la Información, 7(1), 1-31. doi: https://doi.org/10.15517/eci.v7i1.25264

Sandí, J. C. y Cruz, M. A. (2018). Análisis comparativo de juegos móviles educativos basados en posicionamiento. Revista Intersedes, 19(39), 146-170. doi: https://doi.org/10.15517/isucr.v19i39.34075

Sandí, J. C. y Espinoza, I. (2018). Fortalecimiento de la calidad de vida de las personas adultas mayores del Caribe Costarricense. Revista Pensamiento Actual, 18(31), 87-101. Recuperado de https://revistas.ucr.ac.cr/index.php /pensamiento-actual/article/view/35664

Sandí, J. C. y Sanz, C. V. (2018a). Análisis comparativo de juegos serios educativos. Indagación sobre sus posibilidades para la adquisición de competencias tecnológicas en la formación del profesorado. (Tesis de posgrado). Universidad Nacional de La Plata (UNLP), Buenos Aires, Argentina. Recuperado de http://hdl.handle.net/10915/65653

Sandí, J. C. y Sanz, C. V. (2018b). Revisión y análisis sobre competencias tecnológicas esperadas en el profesorado en Iberoamérica. Edutec. Revista Electrónica de Tecnología Educativa, Diciembre (66), 93-121. doi: https://doi.or g/10.21556/edutec.2018.66.1225

Sandí, J. C., Sanz, C. V. y Lovos, E. N. (2018). Juegos serios para la indagación de competencias tecnológicas que puedan integrarse en la práctica pedagógica del profesorado. Una propuesta de aplicación en la Sede del Atlántico de la Universidad de Costa Rica (UCR). (Tesis de maestría). Universidad Nacional de La Plata (UNLP), Buenos Aires, Argentina. Recuperado de http://sedici.unlp.edu.ar/handle/10915/71063

Waag-Society. (2019). Frequency 1550. Recuperado de http://mobilelearningacademy.org/projects/frequency-1550/

Wartenweiler, T. (2018). Serious play in education for social justice - A mixed-methods evaluation. NAER. Journal of New Approaches in Educational Research, 7(1), 61-69. doi: https://doi.org/10.7821/naer.2018.1.260

\section{Notas}

[1] Para efectos de esta investigación, el término tecnológicas refiere a tecnologías digitales. Es importante realizar la aclaración debido a que el concepto de tecnología es más amplio y no se circunscribe únicamente a tecnologías digitales, sino que puede abarcar tecnologías organizativas, tecnologías simbólicas y artefactuales (Sandí y Sanz, 2018b)

\section{BY-NC-ND}

